\title{
The role of levonorgestrel intrauterine systems in the treatment of symptomatic fibroids
}

\author{
Wojciech Wrona, Anna Stępniak, Piotr Czuczwar \\ $3^{\text {rd }}$ Department of Gynaecology, Medical University, Lublin, Poland
}

\begin{abstract}
Uterine fibroids are considered to be the most frequently occurring tumours in females. The majority of fibroids do not require any treatment. When symptomatic, the major ailments include abnormal uterine bleeding, painful menstruation, pelvic pressure or pain, urinary problems, constipation, infertility, and recurrent pregnancy loss. Surgery remains a mainstay of symptomatic uterine fibroids therapy; however, minimally-invasive techniques and pharmacological management have become more available. The levonorgestrel intrauterine system (LNG-IUS) is a T-shaped device with a vertical stem containing a reservoir of levonorgestrel and is widely known for its contraception effect. Moreover, the non-contraceptive benefits of the LNG-IUS have been previously confirmed by numerous studies. LNG-IUS causes reduction of the duration and the amount of menstrual bleeding, with minimal side effects due to release of hormones at the targeted organ. Currently, results from systematic reviews show that LNG-IUS may be an effective and safe treatment for symptomatic uterine fibroids in premenopausal women. However, further studies are required to consolidate the usage of LNG-IUS in the treatment of symptomatic uterine fibroids.
\end{abstract}

Key words: fibroids, intrauterine systems, menorrhagia, levonorgestrel, LNG-IUS.

\section{Introduction}

Fibroids are the most common tumours of the female pelvis [1]. The tumours are benign and occur in approximately $30 \%$ to $50 \%$ of women over 35 years old, and more frequently in black women [2]. The pathogenesis of fibroids remains unclear, although fibroid growth is thought to be related to genetics, steroid hormones, and growth factors [3]. The location and size of fibroids in the uterus are determinants of their clinical manifestations. The vast majority of the tumours are asymptomatic and therefore require no treatment. Bleeding resulting from the presence of fibroids is one of the most commonly reported symptoms in patients with fibroids, and it appears to be associated with histological and hormonal changes in the endometrial tissue that lead to vascular destabilisation of the endometrium and irregular bleeding [4]. Other reported symptoms include pelvic pressure or pain, urinary disorders, constipation, as well as infertility and recurrent pregnancy loss. Thus, both the economic cost and the effect on the quality of life are substantial. Surgery has been the mainstay of fibroid treatment, although various minimally-invasive procedures have been developed, such as uterine artery embolisation, magnetic resonance imaging-guided high-intensity focused ultrasound, laparoscopic occlu- sion of uterine arteries, myolysis, and ulipristal acetate pharmacological treatment. It has been shown that uterine fibroids have significantly increased concentrations of both oestrogen and progesterone receptors compared with normal myometrium [5]. Because fibroids are hormone-dependent tumours their growth is dependent on ovarian activity, especially on oestrogen and progesterone levels, while after the menopause most fibroids shrink [6]. Oestrogen stimulates the growth of fibroids that are exposed to this hormone not only through ovarian steroidogenesis, but also through local conversion of androgens by aromatase within the tumours themselves. The induction of oestrogen receptor $\alpha(E R \alpha)$, causes the augmentation of progesterone receptor (PR) expression and increases the responsiveness of fibroids to progesterone. PR is essential for the response of fibroid tissue to progesterone secreted by the ovaries. Progesterone and PR are crucial for tumour growth, increasing cell proliferation and survival, as well as enhancing extracellular matrix formation. Progesterone has been shown to stimulate the growth of fibroids through a set of key genes, which regulate both apoptosis and proliferation [6, 7]. Most of the available hormonal therapies act on the relevant hormones or their receptors to interfere with either fibroid growth and indirectly with its symptoms or with the symptoms only. 


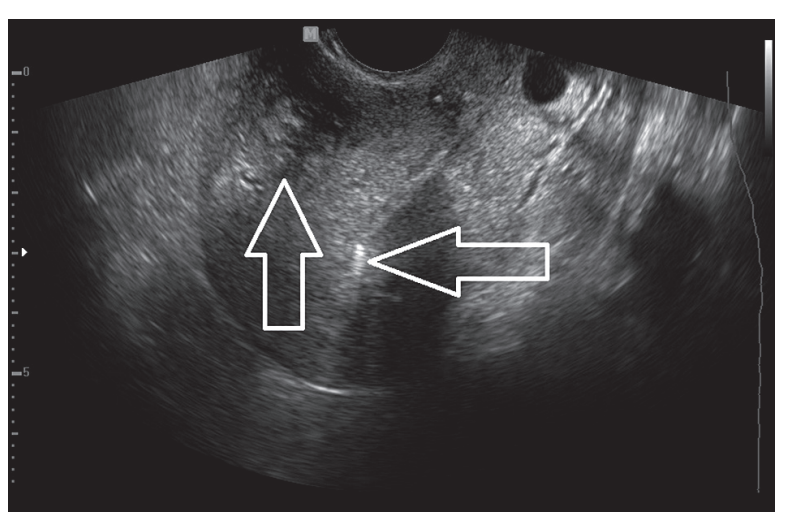

Fig. 1. Ultrasound image of an intramural fibroid (vertical arrow) and a levonorgestrel intrauterine system (horizontal arrow)

Gonadotrophin-releasing hormone (GnRH) analogues were commonly used in the therapy, although their use was limited by the side effects associated with longterm treatment. These analogues were mostly used as a temporising measure in perimenopausal women and in preoperative treatment, due to significant reduction in uterine volume, as well as in fibroid size. Therefore, these compounds helped to restore haemoglobin levels and reduce blood loss during surgery. Their main disadvantage was rebound growth of fibroids upon cessation of therapy, along with the side effects associated with hypoestrogenism, such as climacteric symptoms and loss of bone mass [8]. Selective oestrogen receptor modulators (SERMs), such as raloxifene, were shown to induce fibroid regression only in postmenopausal women, but the therapy had major side effects. Currently, selective progesterone receptor modulators (SPRMs) play a major role in pharmacological treatment of symptomatic fibroids. Moreover, LNG-IUS may be applied in fibroid management. The LNG-IUS is a T-shaped device with a vertical stem containing a reservoir of $52 \mathrm{mg}$ of levonorgestrel, which releases $20 \mu \mathrm{g}$ of levonorgestrel per day and is effective for five years of use, it has been available on the market since the 1990s. An ultrasound image of an LNG-IUS in a patient with fibroids is shown in Fig. 1. LNG-IUS causes a profound suppression of the functional endometrium as well as thickening of the cervical mucous [9]. As a consequence, reduction of the duration and the amount of menstrual bleeding was observed, with minimal side effects. The good safety profile might be explained by the fact that progesterone is released at the targeted organ only. The aim of this article is to review the literature focused on the role of LNG-IUS in the management of symptomatic fibroids.

\section{Liferature review}

Non-contraceptive benefits of the LNG-IUS have been previously confirmed by numerous clinical studies. The strongest evidence, proven by multiple studies in the last decade, shows that LNG-UIS is effective in treat- ing menorrhagia and preventing anaemia. In addition, other benefits for women suffering from symptomatic uterine fibroids have also been reported. Starczewski et al. enlisted the LNG-IUS non-contraceptive benefits including reduction of menstrual blood loss and reduction of uterine volume [10]. Additionally, some researchers reported the decrease of fibroid volume. Tasci et al. observed a statistically significant decrease in fibroid volume $(p=0.04)$, with significant progression of the effect between six and 12 months of treatment [11]. Magalhaes et al. evaluated uterine and fibroid volume in women using LNG-IUS after three years of followup [12]. Even though the fibroid volume remained unchanged, uterine volume showed a significant mean reduction from $156.6 \mathrm{~cm}^{3}$ to $93 \mathrm{~cm}^{3}$ [12]. There is also a case report presenting significant reduction in uterine and fibroid size with the use of the LNG-IUS after one year in a renal transplant patient [13]. In contrast, other studies report no reduction in uterine volume or fibroid size [14]. Both the absence of changes and decrease in fibroid volume are interesting because theoretically it might be expected that progesterone released from the IUS could stimulate fibroid growth [15]. Evidencebased medicine shows that LNG-UIS reduces menstrual blood loss in premenopausal women with uterine fibroids. The mechanisms of LNG-IUS action to reduce menorrhagia and uterine volume are not yet clear. It was suggested that LNG-IUS releases high doses of levonorgestrel into the uterine cavity, causing rapid decidualisation of the endometrial stroma and acting on the uterine vasculature and the receptors in myometrium [16]. The influence of LNG-IUS on fibroid volume has not been unitarily defined in available reviews, and the mechanism of its action remains unclear. Some investigators suggested that progestins might stimulate mitosis within the fibroid and promote its multiplication [17]. Another study indicated that progestin might play a dual role in regulating the growth of fibroid, and that high local concentrations of levonorgestrel might suppress the proliferation of uterine fibroid cells and promote apoptosis [18]. A few studies were conducted to investigate the menstrual blood loss reduction in patients with uterine fibroids. Kriplani et al. assessed the effect of LNG-IUS on uterine and fibroid volume, as well as, the efficacy in reducing menstrual blood loss in fibroid-related menorrhagia. They obtained the following results: one month after LNG-IUS insertion, the Pictorial Blood Loss Assessment Chart (PBAC) score in the fibroid group fell by $86.8 \%(p<0.0001)$. At the $3^{\text {rd }}, 12^{\text {th }}, 24^{\text {th }}, 36^{\text {th }}$, and $48^{\text {th }}$ month, the menstrual blood loss was reduced by $92.1 \%, 97.4 \%, 97.4 \%, 99.5 \%$, and $99.5 \%$, respectively. A similar effect was seen in the idiopathic menorrhagia group. Mean uterine volume was significantly reduced in both groups, but the reduction was greater in patients with fibroids. There was no significant reduction in fibroid volume $(p=0.409)$ [19]. 
Another study, which confirmed the positive effect of LNG-IUS on menorrhagia, also showed that serum levels of haemoglobin, haematocrit, and ferritin increased among LNG-IUS users [20]. Furthermore, Socolov et al. stated that the LNG-IUS is effective in controlling menorrhagia related to the presence of fibroids, but has no significant effect on the size of the tumours [21]. The efficacy of LNG-IUS and low-dose combined oral contraceptive (COC) in reducing fibroid-related menorrhagia was compared in a randomised clinical trial [22]. The reduction of menstrual blood loss was significantly greater in the LNG-IUS group than in the COC group $(90.9 \%$ vs. $13.4 \% ; p<0.001)$. The reduction of PBAC scores was also greater in the LNG-IUS group than in the COC group (88.0\% vs. $53.5 \%$; $p=0.02)$. Moreover, haemoglobin levels increased from $9.7 \mathrm{~g} / \mathrm{dl}$ to $11.7 \mathrm{~g} / \mathrm{dl}(p<0.001)$ and duration of menstrual bleeding decreased from 8.2 days to 1.3 days $(p=0.003)$. The main finding of this study was that LNG-IUS is more effective than $\mathrm{COC}$ in reducing fibroid-related menorrhagia in women with fibroids [22]. A multicentre randomised prospective study found that LNG-IUS markedly reduced the incidence of menorrhagia, fibroids volume, and fibroidrelated surgery in comparison with the copper-IUS [23]. Machado et al. evaluated the percentage of hysterectomies avoided following the insertion of LNG-IUS in perimenopausal women with uterine fibroids and a prior indication for surgery. Within 24 months of follow-up $89.5 \%$ of patients using LNG-IUS avoided hysterectomy and were more satisfied with treatment $(p=0.02) \mathrm{com}$ pared to those treated surgically [24]. Kaunitz et al. suggested that LNG-IUS should be offered to women with fibroids before surgery to reduce bleeding [14]. Recently, a systematic review investigated the efficacy and safety of LNG-IUS as a treatment for symptomatic fibroids. Eleven studies of various designs from the past 10 years were analysed. All studies demonstrated markedly reduced menstrual bleeding. However, changes in uterine and fibroid volume were inconsistent, with some showing a decrease in size and some showing no effect [16].

The usage of LNG-IUS seems to have very few sideeffects such as spotting or irregular menstrual bleeding at the beginning of the therapy. However, the rate of LNG-IUS expulsion was higher among women with uterine fibroids than among those without the tumours. Zapata et al. showed rates of LNG-IUS expulsion in women with uterine fibroids (11\%) and in women without uterine fibroids (3\%) [20]. There are no studies investigating the impact of localisation and size of fibroids on the expulsion rate. The influence of the LNG-IUS on ovarian function has also been assessed. A study by Tasci et al. evaluated the effects of menorrhagia treatment with LNG-IUS on ovarian function, uterine volume, and menstrual cycle. Serum FSH level significantly increased in 69\% of LNG-IUS users $(p=0.021)$. Regression analysis showed significant as- sociation between FSH level and the age of a patient $(p=0.001)$. No significant differences in serum oestradiol and LH levels were found after 12 months follow-up ( $p=0.339$ and $p=0.556$, respectively). After one year, amenorrhoea or hypomenorrhoea developed in $67.4 \%$ of the patients. There were no statistically significant differences in ovarian and uterine volume. The main results of this study demonstrated successful menorrhagia treatment with LNG-IUS without any significant impairment of ovarian function [11].

LNG-IUS is FDA approved for long-acting reversible contraception and treatment of heavy menstrual bleeding. It is also one of the least expensive hormonal medications when the cost is averaged over the five years of recommended use per device. The most common adverse effects reported in the literature are irregular bleeding and ovarian cysts, which resolved spontaneously in time. A Cochrane Collaboration review claims that there is insufficient evidence to support the use of progestogens or progestogen-releasing intrauterine systems in treating premenopausal women with uterine fibroids [25]. On the other hand, a more recent systematic review stressed that LNG-IUS could be a recommended treatment for menorrhagia induced by uterine fibroids [26].

\section{Conclusions}

LNG-IUS is not only a reasonable option for patients desiring long-term contraception, but may also be beneficial in the management of symptomatic uterine fibroids. Systematic reviews show that LNG-IUS may be an effective and safe treatment option for symptomatic uterine fibroids in premenopausal women. There is strong evidence that treatment with LNG-IUS reduces menstrual blood loss and increases blood haemoglobin, ferritin, and haematocrit levels. The safety of the device is high with no adverse effects on ovarian function except for increased risk of ovarian cysts. Moreover, some studies showed a reduction in uterine fibroids size, which could be an interesting field for future research. Further studies are required to consolidate the usage of LNG-UIS in the treatment of symptomatic uterine fibroids.

\section{Disclosure}

Authors report no conflict of interest.

\section{References}

1. Buttram VC. Uterine leiomyomata--aetiology, symptomatology and management. Prog Clin Biol Res 1986; 225: 275-296.

2. Baird DD, Dunson DB, Hill MC, et al. High cumulative incidence of uterine leiomyoma in black and white women: ultrasound evidence. Am J Obstet Gynecol 2003; 188: 1: 100-107.

3. Eggert SL, Huyck KL, Somasundaram P, et al. Genome-wide Linkage and Association Analyses Implicate FASN in Predisposition to Uterine Leiomyomata. Am J Hum Genet 2012; 91: 621-628. 
4. Maia H, Pimentel K, Casoy J, et al. Aromatase expression in the eutopic endometrium of myomatous uteri: The influence of the menstrual cycle and oral contraceptive use. Gynecol Endocrinol 2007; 23: 320-324.

5. Tamaya T, Fujimoto J, Okada H. Comparison of cellular levels of steroid receptors in uterine leiomyoma and myometrium. Acta Obstet Gynecol Scand 1985; 64: 307-309.

6. Bulun SE. Uterine fibroids. N Engl J Med 2013; 369: 1344-1355.

7. Moravek MB, Yin P, Ono M, et al. Ovarian steroids, stem cells and uterine leiomyoma: therapeutic implications. Hum Reprod Update 2015; 21: 1-12.

8. Murillo EO, Cano A. Análogos de la GnRH en el tratamiento de los miomas. Med Clin (Barc) 2013; 141: 35-39.

9. Bayer LL, Hillard PJA. Use of levonorgestrel intrauterine system for medical indications in adolescents. J Adolesc Heal 2013; 52: S54-S58..

10. Starczewski A, Iwanicki M. Intrauterine therapy with levonorgestrel releasing IUD of women with hypermenorrhea secondary to uterine fibroids. Ginekol Pol 2000; 71: 1221-1225.

11. Tasci Y, Caglar GS, Kayikcioglu F, et al. Treatment of menorrhagia with the levonorgestrel releasing intrauterine system: effects on ovarian function and uterus. Arch Gynecol Obstet 2009; 280: 39-42.

12. Magalhães J, Aldrighi JM, de Lima GR. Uterine volume and menstrual patterns in users of the levonorgestrel-releasing intrauterine system with idiopathic menorrhagia or menorrhagia due to leiomyomas. Contraception 2007; 75: 193-198.

13. Fong YF, Singh K. Effect of the levonorgestrel-releasing intrauterine system on uterine myomas in a renal transplant patient. Contraception 1999; 60: 51-53.

14. Kaunitz AM. Progestin-releasing intrauterine systems and leiomyoma. Contraception 2007; 75: S130-S133.

15. Talaulikar VS, Manyonda I. Progesterone and progesterone receptor modulators in the management of symptomatic uterine fibroids. Eur J Obstet Gynecol Reprod Biol 2012; 165: 135-140

16. Jiang $\mathrm{W}$, Shen $\mathrm{Q}$ Chen $\mathrm{M}$, et al. Levonorgestrel-releasing intrauterine system use in premenopausal women with symptomatic uterine leiomyoma: A systematic review. Steroids 2014; 86: 69-78.

17. Pavlovich SV, Volkov NI, Burlev VA. Proliferative activity and level of steroid hormone receptors in the myometrium and myoma nodes in different phases of menstrual cycle. Bull Exp Biol Med 2003; 136: 396-398.

18. Xu Q, Qiu L, Zhu L, et al. Levonorgestrel inhibits proliferation and induces apoptosis in uterine leiomyoma cells. Contraception 2010; 82: 301-308.

19. Kriplani A, Awasthi D, Kulshrestha V, et al. Efficacy of the levonorgestrelreleasing intrauterine system in uterine leiomyoma. Int J Gynecol Obstet 2012; 116: 35-38.

20. Zapata LB, Whiteman MK, Tepper NK, et al. Intrauterine device use among women with uterine fibroids: a systematic review. Contraception 2010; 82: 41-55.

21. Socolov D, Blidaru I, Tamba B, et al. Levonorgestrel releasing-intrauterine system for the treatment of menorrhagia and/or frequent irregular uterine bleeding associated with uterine leiomyoma. Eur J Contracept Reprod Heal Care 2011; 16: 480-487.

22. Sayed GH, Zakherah MS, El-Nashar SA, et al. A randomized clinical trial of a levonorgestrel-releasing intrauterine system and a low-dose combined oral contraceptive for fibroid-related menorrhagia. Int I Gynecol Obstet 2011; 112: 126-130.

23. Sivin I, Stern J. Health during prolonged use of levonorgestrel 20 micrograms/d and the copper TCu 380Ag intrauterine contraceptive devices: a multicenter study. International Committee for Contraception Research (ICCR). Fertil Steril 1994; 61: 70-77.

24. Machado RB, de Souza IM, Beltrame A, et al. The levonorgestrel-releasing intrauterine system: its effect on the number of hysterectomies performed in perimenopausal women with uterine fibroids. Gynecol Endocrinol 2013; 29: 492-495.

25. Sangkomkamhang US, Lumbiganon P, Laopaiboon M, et al. Progestogens or progestogen-releasing intrauterine systems for uterine fibroids. Cochrane Database Syst Rev 2013; (2): CD008994.

26. Bartels CB, Cayton KC, Chuong FS, et al. An Evidence-based approach to the medical management of fibroids. Clin Obstet Gynecol 2016; 59: $30-52$ 